\title{
Comunicación
}

\section{Presencia de Piscirickettsia salmonis en Truchas de Cultivo (Oncorhynchus mykiss) en Junín, Perú}

\author{
Presence of Piscirickettsia salmonis in Farmed Trout (Oncorhynchus mykiss) in \\ Junin, Peru \\ Jefferson Yunis A. ${ }^{1,3}$, Jahir Anicama D. ${ }^{1}$, Alberto Manchego S. ${ }^{2}$, Nieves Sandoval C. ${ }^{1}$
}

\section{RESUMEN}

\begin{abstract}
El objetivo de este estudio fue determinar la posible presencia de Piscirickettsia salmonis en truchas de cultivo (Oncorhynchus mykiss) en la cuenca del río Mantaro, Junín, Perú, mediante la prueba de inmunofluorescencia indirecta (IFI). Se obtuvieron 180 truchas en forma aleatoria de tres piscigranjas, se beneficiaron, se les realizó la necropsia y se recolectaron muestras de riñón y bazo. Los tejidos fueron sometidos a la prueba de inmunofluorescencia indirecta (IFI). Todas las muestras resultaron negativas a $P$. salmonis; sin embargo, se encontraron peces con signos clínicos de enfermedad como úlceras en la piel, melanosis, abultamiento del abdomen y exoftalmia; asimismo, en órganos internos se encontraron hemorragias petequiales en hígado, ciegos pilóricos y grasa abdominal, esplenomegalia y palidez hepática.
\end{abstract}

Palabras clave: Piscirickettsia salmonis; Oncorhynchus mykiss; Junín; inmunofluorescencia indirecta

\section{Abstract}

This study aimed to determine the possible presence of Piscirickettsia salmonis in farmed rainbow trout (Oncorhynchus mykiss) in the Mantaro river basin, Junin, Peru, by the indirect inmunofluorescence test (IFAT). Kidney and spleen samples were collected from 180 trout from three fish farms. All samples resulted negative to $P$. salmonis; however, some individuals showed clinical sings of disease as skin ulcer, melanosis, abdominal

\footnotetext{
${ }^{1}$ Laboratorio de Histología, Embriología y Patología Veterinaria, ${ }^{2}$ Laboratorio de Microbiología y Parasitología Veterinaria, Facultad de Medicina Veterinaria, Universidad Nacional Mayor de San Marcos, Lima, Perú

${ }^{3}$ E-mail:jefyunis@gmail.com
}

Recibido: 20 de enero de 2014

Aceptado para publicación: 25 de setiembre de 2014 
swelling and exophthalmoses. Also, in the internal organs was found petechial hemorrhage in liver, pyloric caeca and spleen, splenomegaly and pale liver.

Key words: Piscirickettsia salmonis; Oncorhynchus mykiss; Junin; indirect immunofluorescence

\section{INTRODUCCIÓN}

La trucha arcoíris (Oncorhynchus mykiss) es la especie dulceacuícola más importante en el Perú, especialmente en las zonas altoandinas. La región Junín, en la Sierra Central, destaca por la producción de truchas, llegando a producir de 1337 toneladas en 2003 a 2078 toneladas en 2008 (FAO, 2012).

Este crecimiento debe sustentarse en adecuadas prácticas sanitarias, ya que estas influyen directamente sobre los índices productivos y reproductivos de los peces. Entre las enfermedades más comunes en los principales países productores de trucha, se encuentra la piscirrickettsiosis, conocida también como «síndrome rickettsial salmonídeo» (SRS) (Lannan et al., 1999).

El agente etiológico, identificado como Piscirickettsia salmonis, corresponde a un microorganismo gram negativo, no móvil, no encapsulado, habitualmente cocoide, en pares o en forma de anillo (Lannan et al., 1999). Es una bacteria patógena intracelular obligada y se agrupa en la subdivisión gamma de las proteobacterias (Lannan et al., 1999; Austin B y Austin D, 2007).

La piscirrickettsiosis es una enfermedad de transmisión vertical y horizontal. Los peces infectados presentan letargia, nado superficial y oscurecimiento de la piel. También es común observar anorexia y lesiones en la piel, desde pequeñas áreas de escamas elevadas hasta úlceras superficiales (Lannan et al, 1999).
En la necropsia es común encontrar inflamación en los riñones, esplenomegalia, anemia, ascitis, lesiones en el hígado y hemorragias petequiales en el estómago, intestino, vejiga natatoria, musculatura del cuerpo y grasa visceral (House et al., 1999; OIE, 2003). En el hígado se pueden observar áreas grises y amarillentas, a manera de un puntilleo o en forma de anillos en los peces con enfermedad crónica (Lannan et al., 1999; OIE, 2003). En algunos casos se puede observar un material mucoide amarillento en la luz del intestino (Austin B y Austin D, 2007).

Para el diagnóstico se disponen de métodos serológicos, inmunohistoquímicos y moleculares (Lannan et al., 1999). La confirmación de $P$. salmonis en el cultivo celular puede hacerse mediante las pruebas de inmunofluorescencia indirecta con anticuerpo (IFI) o por PCR (OIE, 2003). La técnica de IFI ha resultado ser una técnica sensible y específica para este organismo (OIE, 2006).

En el Perú, el agente Piscirrickettsia salmonis, causante de esta enfermedad, representa un riesgo para la crianza de trucha arcoíris debido a la constante importación de ovas, que podría permitir el ingreso de esta enfermedad al país y ocasionar problemas y pérdidas económicas. No existen estudios preliminares específicos acerca de esta bacteria en el país, por lo que el objetivo de este estudio fue determinar la frecuencia de este agente en la cuenca del Mantaro, una de las principales zonas productoras de truchas del Perú. 


\section{Materiales y Métodos}

\section{Lugar de Estudio}

La colección de muestras se realizó en tres piscigranjas del departamento de Junín, Perú, localizadas a $3400 \mathrm{msnm}$. Se trabajó en las piscigranjas El Edén y Arco Iris en febrero de 2009, que corresponde a la época de lluvias, con una temperatura ambiental de 10 a $18^{\circ} \mathrm{C}$, y en la piscigranja Rumihuasi en agosto de 2009, que corresponde a la época seca, con una temperatura ambiental de -1 a $13{ }^{\circ} \mathrm{C}$. Edén y Arco Iris tienen una producción anual de 50 y 25 toneladas métricas, respectivamente, y abastecen el mercado local y supermercados de la ciudad de Lima, en tanto que Rumihuasi tiene una producción de 53 toneladas métricas y solo abastece el mercado local.

El procesamiento de las muestras se realizó en el Laboratorio de Ictiopatología de la Facultad de Medicina Veterinaria, Universidad Nacional Mayor de San Marcos, Lima.

\section{Tamaño de Muestra}

En el cálculo del número muestral se siguieron las indicaciones del Manual de Animales Acuáticos de la OIE (2006), donde asumiendo una prevalencia de $1 \%$ y un tamaño de lote mayor de 2000 peces, se colectaron un mínimo de 60 truchas por piscigranja.

\section{Obtención de Muestras}

Las truchas se colectaron de varias pozas que contenían los peces juveniles ( $40.0 \pm$ $7.5 \mathrm{~g}$ de peso y $17.0 \pm 4 \mathrm{~cm}$ de largo) de aproximadamente 4 meses de edad. El sacrificio se realizó mediante la técnica de corte medular con hoja de bisturí, entre el cerebro y médula espinal (Rosenthal, 2007).

La necropsia se llevó a cabo de acuerdo a Ostrande (2000). Se dejó expuestas las vísceras in situ para proceder a la toma de las muestras. Se tomaron cortes de tejido renal y esplénico, de 5 a $10 \mathrm{~mm}$, de cada individuo, se realizó la impronta en láminas portaobjeto y se fijó en acetona por $10 \mathrm{~min}$.

Se hicieron mediciones de temperatura (termómetro de alcohol), contenido de oxígeno (kit Sera ${ }^{\circledR}$ Test O2, Heinsberg, Alemania) y pH (kit Sera ${ }^{\circledR}$ Test pH, Heinsberg, Alemania) del agua de las pozas.

\section{Detección de la Bacteria por IFI}

Se emplearon las improntas de bazo y riñón en la prueba de IFI, utilizando el kit comercial SRS Fluorotest (Laboratorios BiosChile, 2008). Como control positivo, se utilizaron láminas fijadas en xilol con la cepa LF-89 de Piscirickettsia salmonis, procedente del Laboratorio de Patobiología de la Facultad de Medicina Veterinaria de la Universidad de Chile.

\section{Resultados}

Entre los principales signos clínicos observados en los peces se encontraron melanosis $(24.4 \%)$, abdomen abultado (10.6\%), exoftalmia (8.3\%) y úlceras en la piel $(7.8 \%)$. Además, se observaron peces con nado errático y superficial. En la evaluación de los órganos internos se observó palidez hepática $(22.8 \%)$, esplenomegalia (13.3\%) y focos hemorrágicos en hígado, in-

Cuadro 1. Características del agua de las pozas de crianza de trucha arcoíris en tres piscigranjas de la zona de Junín, Perú

\begin{tabular}{lccc}
\hline Piscigranja & $\begin{array}{c}\text { Temperatura } \\
\left({ }^{\circ} \mathrm{C}\right)\end{array}$ & $\begin{array}{c}\text { Oxígeno } \\
(\mathrm{mg} / \mathrm{L})\end{array}$ & $\mathrm{pH}$ \\
\hline El Edén & 12 & 8.0 & 6.8 \\
Arco iris & 11 & 4.7 & 7.9 \\
Rumihuasi & 12 & 4.5 & 7.5 \\
\hline
\end{tabular}


(A)

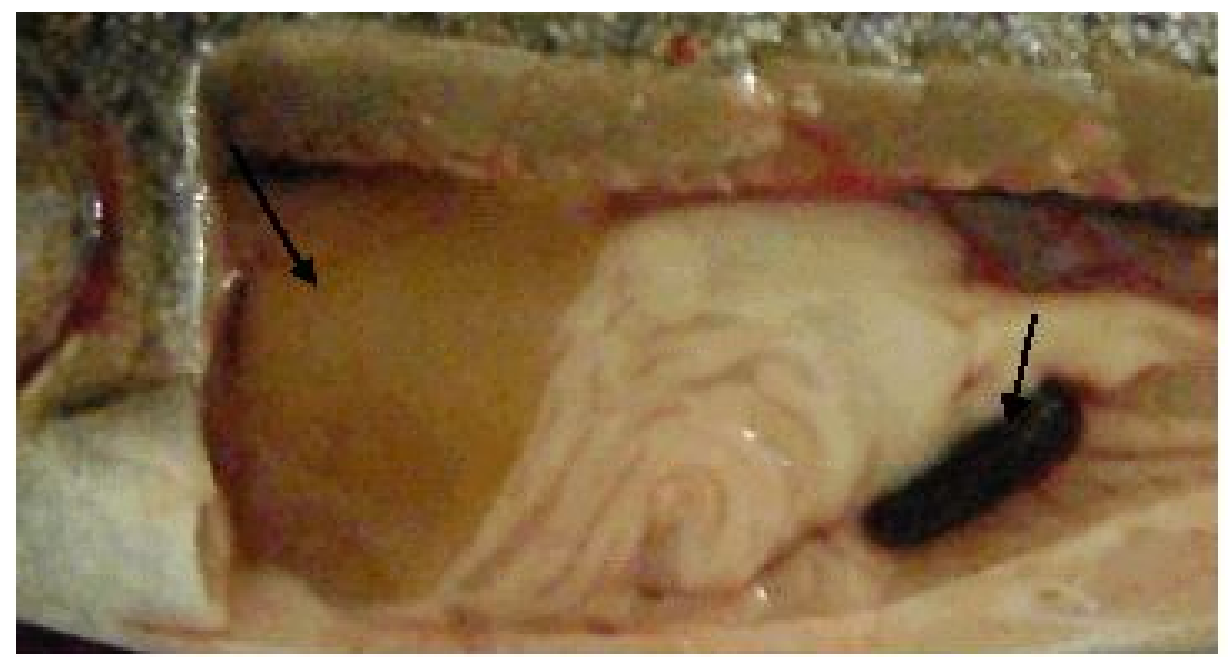

(B)

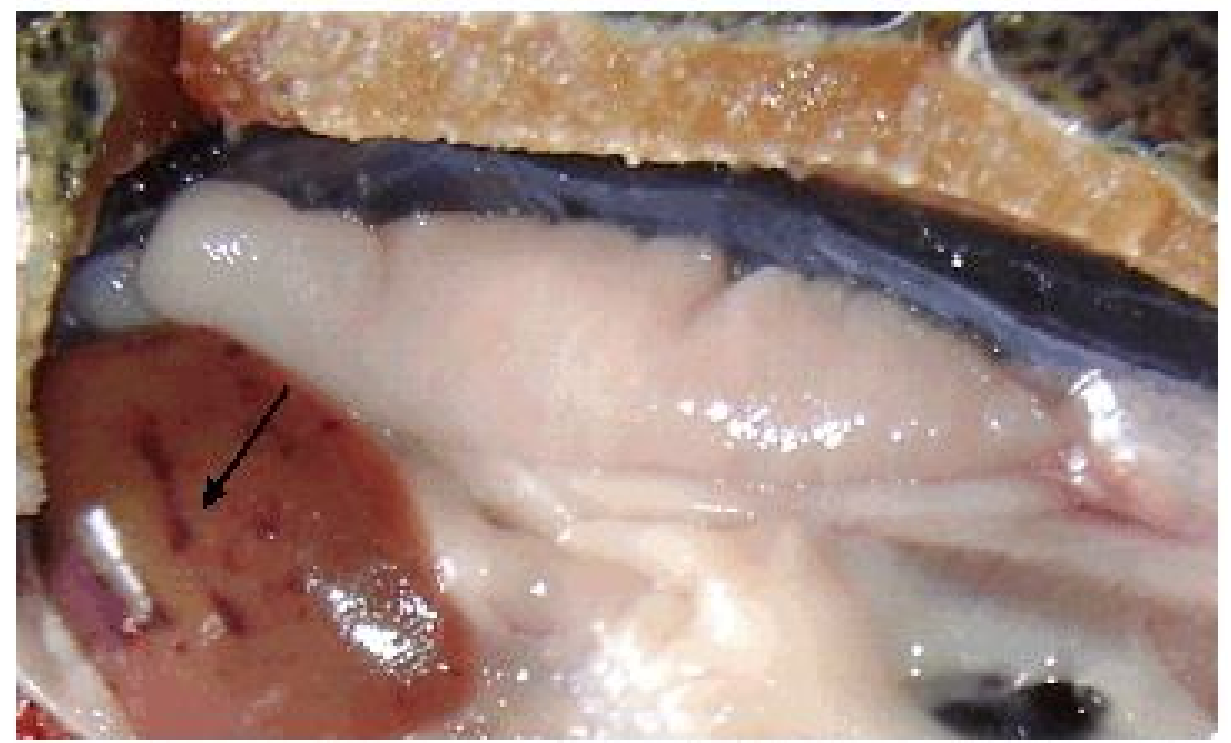

(C)

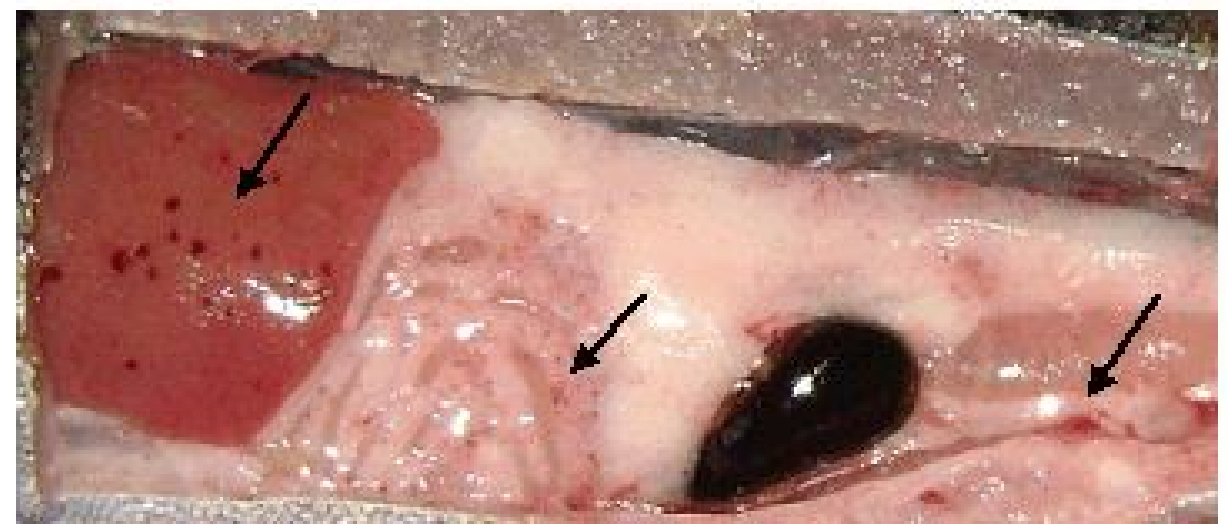

Figura 1. Lesiones en órganos internos en truchas arcoíris. (A) Palidez hepática y esplenomegalia; (B) Focos hemorrágicos en hígado; (C) Hemorragia petequial en hígado, ciegos pilóricos y grasa abdominal 
testino, ciego pilórico y grasa peritoneal (13.9\%) (Fig. 1). No obstante, pese a observarse signos clínicos sugerentes de la enfermedad, no se encontraron muestras positivas a Piscirickettsia salmonis mediante la prueba de IFI.

Las características del agua de las pozas en las piscigranjas se muestran en el Cuadro 1. Asimismo, cabe resaltar la presencia de abundante material en suspensión en el agua en las tres piscigranjas.

\section{Discusión}

En la mayoría de las enfermedades infecciosas y no infecciosas en los peces de cultivo se presentan signos clínicos y lesiones de tipo general, lo que hace difícil llegar a un diagnóstico clínico definitivo. En el presente estudio se hallaron peces con lesiones sugerentes de piscirickettsiosis, pero ninguno de estos casos fue positivo a esta enfermedad. Cuadros clínicos similares fueron obtenidos por Fryer et al. (1992) y Smith et al. (1999) en más del 90\% de los peces al inocular P. salmonis en truchas arcoíris. Asimismo, en infecciones naturales en salmón coho (Oncorhynchus kisutch) con P. salmonis se han encontrado lesiones similares, pero afectando gran cantidad de peces (Branson y Nieto, 1991; Cvitanich et al., 1991; Garces et al., 1991). Por otro lado, Olsen et al. (1997) encontraron lesiones similares en salmones del atlántico juveniles, con una frecuencia menor del $1 \%$, pudiendo identificar a $P$. salmonis por IFI y por cultivo celular.

La presencia de abundante material en suspensión en el agua y la baja concentración de oxígeno en las piscigranjas Arco iris y Rumihuasi se convierten en potenciales factores de riesgo para el incremento de la susceptibilidad de las truchas a agentes infecciosos, las cuales podrían haber facilitado la ocurrencia de lesiones y signos clínicos encontrados. Por otro lado, los resultados negativos por IFI a $P$. salmonis sugieren que la enfermedad no se encuentra presente en esta zona o se encuentra en una prevalencia muy baja.

\section{Literatura Citada}

1. Austin B, Austin D. 2007. Characteristics of the disease, Pisciricketsiaceae representative: Piscirickettsia salmonis. En: Bacterial fish pathogens. Disease of farmed and wild fish. $4^{\text {th }}$ ed. UK: Praxis Publishing. p 81-148.

2. Branson E, Nieto D. 1991. Description of a new disease condition occurring in farmed coho salmon, Oncorhynchus kisutch (Walbaum), in South America. J Fish Dis 14: 147-156. doi: 10.1111/ j.1365-2761.1991.tb00585.x

3. Cvitanich J, Garate O, Smith C. 1991. The isolation of rickettsia-like organism causing disease and mortality in Chilean salmonids and its confirmation by Koch's postulate. J Fish Dis 14: 121-145. doi: 10.1111/j.1365-2761.1991.tb00584.x

4. [FAO] Organización de las Naciones Unidas para la Alimentación y la Agricultura. 2012. El estado mundial de la pesca y la acuicultura (SOFIA). [Internet]. Disponible en: http:// www.fao.org/fishery/sofia/es

5. Fryer J, Lannan C, Giovannoni S, Wood N. 1992. Piscirickettsia salmonis gen. nov., sp. nov., the causative agent of an epizootic disease in salmonid fishes. Int J Syst Bacteriol 42: 120-126.

6. Garcés L, Larenas $P$, Smith $P$, Sandino S, Lannan C, Fryer L. 1991. Infectivity of a rickettsia isolated from coho salmon Oncorhynchus kisutch. Dis Aquat Organ 11: 93-97.

7. House M, Bartholomew J, Winton J, Fryer J. 1999. Relative virulence of three isolates of Piscirickettsia salmonis for coho salmon Oncorhynchus kisutch. Dis Aquat Organ 35: $107-$ 113. doi: 10.3354/dao035107 
8. Laboratorios BiosChile. 2008. Manual: SRS - Fluorotest indirecto. Santiago de Chile: BiosChile. 8 p.

9. Lannan CN, Bartholomew JL, Fryer JL. 1999. Rickettsial and chlamydial infections in fish. In: Woo PTK, Bruno DW (eds). Fish disease and disorders. Vol. 3. Viral bacterial and fungal infections. p 245-267.

10. [OIE] Organización Mundial de Sanidad Animal. 2003. Piscirickettsiosis. Manual of diagnostic tests for aquatic animals. Ch 2.1.13. $4^{\text {th }}$ ed. Paris: OIE. $358 \mathrm{p}$.

11. [OIE] Organización Mundial de Sanidad Animal. 2006. Manual de pruebas de diagnóstico para los animales acuáticos. $5^{\text {th }}$ ed. París: OIE. 384 p.
12. Olsen AB, Melby HP, Spielberg L, Evensen Ø, Hastein T. 1997. Piscirrickettsia salmonis infection in Atlantic salmon, Salmo salar, in Norway. Epidemiological, pathological and microbiological findings. Dis Aquat Organ 31: 35-48.

13. Ostrande GK. 2000. The laboratory fish. San Diego, USA: Academic Press. 678 p.

14. Rosenthal S. 2007. Frecuencia de lipidosis hepática en trucha arcoíris (Oncorhynchus mykiss) de fase juvenil en una piscigranja de la sierra central del Perú. Tesis de Médico Veterinario. Lima: Univ Nacional Mayor de San Marcos. 74 p.

15. Smith P, Pizarro P, Ojeda P, Contreras J, Oyanedel S, Larenas J. 1999. Routes of entry of Piscirickettsia salmonis in rainbow trout Oncorhyn-chus mykiss. Dis Aquat Organ 37: 165-172. 